\title{
The weighted curvature approximation in scattering from sea surfaces
}

\author{
Charles-Antoine Guérin $^{\mathrm{a},{ }^{*}}$; Gabriel Soriano ${ }^{\mathrm{b}}$; Bertrand Chapron ${ }^{\mathrm{c}}$ \\ a LSEET, CNRS/Université du Sud-Toulon-Var, La Garde, France ; \\ ${ }^{\mathrm{b}}$ Institut Fresnel, CNRS/Université Paul Cézanne, Marseille France ; \\ c Laboratoire d'Océanographie Spatiale, IFREMER, Plouzané, France; \\ *: Corresponding author : C.-A. Guérin, email address : querin@univ-tln.fr
}

\begin{abstract}
:
A family of unified models in scattering from rough surfaces is based on local corrections of the tangent plane approximation through higher-order derivatives of the surface. We revisit these methods in a common framework when the correction is limited to the curvature, that is essentially the secondorder derivative. The resulting expression is formally identical to the weighted curvature approximation, with several admissible kernels, however. For sea surfaces under the Gaussian assumption, we show that the weighted curvature approximation reduces to a universal and simple expression for the off-specular normalized radar cross-section (NRCS), regardless of the chosen kernel. The formula involves merely the sum of the NRCS in the classical Kirchhoff approximation and the NRCS in the small perturbation method, except that the Bragg kernel in the latter has to be replaced by the difference of a Bragg and a Kirchhoff kernel. This result is consistently compared with the resonant curvature approximation. Some numerical comparisons with the method of moments and other classical approximate methods are performed at various bands and sea states. For the copolarized components, the weighted curvature approximation is found numerically very close to the cut-off invariant two-scale model, while bringing substantial improvement to both the Kirchhoff and small-slope approximation. However, the model is unable to predict cross-polarization in the plane of incidence. The simplicity of the formulation opens new perspectives in sea state inversion from remote sensing data.
\end{abstract}




\section{Introduction}

Despite the ever increasing computer facilities, the exact computation of the field scattered from the sea surface is still a challenging problem, especially in the microwave regime where the required number sampling point becomes prohibitive. Thus, the simulation of sea radar return strongly relies on approximate methods, whose efficiency and accuracy has been constantly improving over recent years (e.g. [1]). To cope with both fundamental limits, namely Geometrical Optics (GO) and the Small Perturbation Method (SPM), various unified models have been proposed on the basis of local curvature corrections to the Kirchhoff Approximation (KA). Among the wealth of existing models, there is a family of methods which proceed to find directly the scattering amplitude from some general physical constraints the latter should satisfy. These methods are essentially due to the impulse of Tanos Elfouhaily, inspired by earlier works of Dashen and Wurmser. They are:

1. the Local Weight Approximation (LWA) [2, 3, 4]

2. the Resonant Curvature Approximation (RCA) $[5,6,7]$

3. the Weighted Curvature Approximation (WCA) $[8,9,10]$

The first method is based on tilt invariance properties of the scattering amplitude and is shown to be of the accuracy of the square of the curvature. Its main limitation, however, is its restriction to the perfectly conducting case. The last two methods are purely heuristic as they postulate the form of the desired kernel, but satisfy the correct asymptotic limits and the tilt invariance at first-order in slope. The formalism is common to the dielectric and perfectly conducting case. The purpose of this paper is to revisit the last method in the framework of random rough surfaces with slow fall-off of the power spectrum. This typically applies to wind waves surfaces, which exhibit power-law spectra over several decades. In the microwave regime, such surfaces are truly multiscale since the electromagnetic wavelength lies between the large gravity waves and the small capillary ripples. This scattering problem is therefore out of the scope of both the classical KA (which is known to operate well on the large-scale components only) and SPM (which describes Bragg scattering by the small ripples) and is classically addressed by the Two-Scale Model (TSM). As it performs a dynamical transition between the two limiting cases (KA and SPM), the WCA can, in principle, cope with both small and large scales, and is expected to be close to the TSM. However, in its original formulation, WCA has been found quite complex from both a theoretical and numerical point of view.

We will show how the WCA can be more satisfactorily justified by a systematic treatment of curvature corrections and, at the same time, considerably simplified. By curvature correction it is essentially meant a term involving second-order derivatives of the surface, which should account for the deviation 
of the latter from its tangent plane over a distance comparable to the illuminating wavelength. As we will see, a curvature correction for the statistical normalized radar cross-section (NRCS) under the Gaussian assumption typically brings in a Laplacian of the correlation function under a Kirchhoff integral. By "Kirchhoff integral" we denote the classical integral which is ubiquitous in approximated NRCS from rough surfaces, starting with the Kirchhoff or tangent plane approximation.

The key point is that a Kirchhoff integral operates as a filter around the Bragg frequency $\left(Q_{H}\right.$ in the following). For sea-spectra we demonstrate what we will call the "Kirchhoff filtering formula":

$$
\frac{1}{(2 \pi)^{2}} \int \boldsymbol{d} \boldsymbol{r} \mathrm{e}^{-\mathrm{i} \boldsymbol{Q}_{H} \cdot \boldsymbol{r}} \mathrm{e}^{-Q_{z}^{2}\left(\rho_{0}-\rho\right)} \Delta \rho \simeq-Q_{H}^{2} \Gamma\left(\boldsymbol{Q}_{H}\right),
$$

where $\Gamma$ is the power spectrum of the surface, $\rho$ its correlation function, and $\boldsymbol{Q}_{H}$ and $Q_{z}$ the horizontal and vertical components of the Ewald vector, respectively. The dependence on the space variable $\boldsymbol{r}=(x, y)$ in the correlation function is implicitly assumed and the same holds for its Laplacian $\Delta \rho=\partial_{x x} \rho+\partial_{y y} \rho$. The naught subscript for a function indicates its value at the origin $\left(\rho_{0}=\rho(0)\right)$.

We will show this formula to hold away from a specular region controlled by the mean square slope (mss) of the surface: $Q_{H}>>\sqrt{m s s} Q_{z}$. Strange as it may seem, the curvature-corrected Kirchhoff integral (1.1) takes the same limiting form in the high-frequency limit $\left(Q_{z}^{2} \rho_{0} \rightarrow+\infty\right)$ as in the small-perturbation limit $\left(Q_{z}^{2} \rho_{0} \rightarrow 0\right)$. In virtue of this mathematical property, Kirchhoff integrals and power spectra at the Bragg frequency can constitute the elementary building blocks of many unified approximate scattering models. Hence, the integral (1.1) can be seen as a filtered curvature around the resonant frequency, to fulfill our expectation of a deviation from the tangent plane at the scale of the wavelength.

In the present work we propose a simple way to establish local curvature corrections to the KA. We show that they can be realized though a kernel which is quadratic in slope, in which case they become formally identical to the Weighted Curvature Approximation. We then use the fundamental property of the Kirchhoff filter to derive an universal and simple formula for the incoherent NRCS, common to all curvature kernels (formula (4.32)). The formula involves merely the sum of the NRCS in the classical Kirchhoff approximation and the NRCS in the Small Perturbation Method, except that the Bragg kernel in the latter has to be replaced by the difference of a Bragg and a Kirchhoff kernel. This expression is valid for large-scale, ripple-like as well as broadband surfaces, although it is non-trivial in the latter case only. As a byproduct, the hidden link with the Resonant Curvature Approximation is unveiled. Some numerical comparisons with the Method of Moments and other classical approximate methods are performed. In spite of its simplicity, the Weighted Curvature Approximation is found to bring substantial improvement over the classical methods of the same level of complexity, namely KA and the first-order Small-Slope Approximation (SSA1). Numerical comparisons also show a good agreement with the cutoff invariant Two-Scale Model [11], even though there is no explicit separation 
of scales in the technique. We conclude with some general considerations on potential applications of this result to sea spectra inversion procedures.

\section{A simple approach to the Weighted Curvature Approximation}

Consider a rough surface $z=\eta(\boldsymbol{r})$ with mean horizontal plane $\boldsymbol{r}=(x, y)$ and upward directed vertical axis, separating the vacuum (upper medium) from a dielectric or conducting homogeneous medium (lower medium). An incoming, downward propagating, harmonic plane wave with wave vector $\boldsymbol{K}_{0}$ illuminates the surface. The scattering amplitude $\mathbb{S}$ expresses the scattered electric field along the upwards wave vector $\boldsymbol{K}$ (we refer to [1] for the exact definition). It is convenient to introduce the Ewald vector $\boldsymbol{Q}=\boldsymbol{K}-\boldsymbol{K}_{0}$, together with its horizontal and vertical projections $\boldsymbol{Q}_{H}$ and $Q_{z}$, respectively. The Kirchhoff or tangent plane solution,

$$
\mathbb{S}=\frac{\mathbb{K}}{Q_{z}} \int \frac{d r}{(2 \pi)^{2}} \mathrm{e}^{-\mathrm{i} Q_{H} \cdot r} \mathrm{e}^{-\mathrm{i} Q_{z} \eta},
$$

is known to be exact for tilted planes, that is for surfaces with vanishing higherorder $(\geq 2)$ derivatives. The Kirchhoff dyad $\mathbb{K}$ depends solely on the scattering vectors $\left(\boldsymbol{K}, \boldsymbol{K}_{0}\right)$ and the permittivity of the lower medium. It can be found explicitely in the appendix of [1] for different boundary conditions at the interface. We recall that the dyads can be represented by four matrix elements after projection on a polarization basis. For smooth but non planar surfaces, a correction to the Kirchhoff approximation can be sought in the form:

$$
\mathbb{S}=\frac{1}{Q_{z}} \int \frac{\boldsymbol{d} \boldsymbol{r}}{(2 \pi)^{2}}\left(\mathbb{K}+\frac{\mathrm{i}}{Q_{z}} \mathbb{T}(\boldsymbol{\nabla})[\eta]\right) \mathrm{e}^{-\mathrm{i} \boldsymbol{Q}_{H} \cdot \boldsymbol{r}} \mathrm{e}^{-\mathrm{i} Q_{z} \eta},
$$

where the notation $\mathbb{T}(\boldsymbol{\nabla})[\eta]$ indicates a tensor-valued differential operator of the surface $\eta$. Although this operator also depends on the scattering vectors $\left(\boldsymbol{K}, \boldsymbol{K}_{0}\right)$ we will omit this obvious dependence to keep reasonably simple notations. We will search for a local correction to the tangent plane, that is a functional $\mathbb{T}$ which involves only the shape of the surface at the point under consideration. Such a functional can be built by a general expansion in order of derivatives:

$$
\mathbb{T}(\boldsymbol{\nabla})[\eta]=\sum_{1 \leq n+m \leq N} \mathbb{T}_{n m} \partial_{x}^{n} \partial_{y}^{m} \eta
$$

where the dyads $\mathbb{T}_{n m}$ are roughness independent. Such a series is in principle infinite but a systematic treatment of derivatives of arbitrary order in a Kirchhoff integral seems out of reach. Therefore, we chosed to limit ourselves to the lowest-order correction to the tangent plane approximation, namely secondorder derivatives. This amounts to make a parabolic rather than facet approximation to the surface. While first-order derivatives of the surface are clearly identified with slopes, the meaning of second-order derivatives is less obvious. 
However, recalling that the curvature $\gamma$ of a one-dimensional surface $\eta$ is expressed by:

$$
\gamma=\frac{\eta^{\prime \prime}}{\left(1+\left(\eta^{\prime}\right)^{2}\right)^{3 / 2}}
$$

we can interpret second-order derivatives as surface curvature as long as the slopes remain small, which is the case for the sea surface. Now, any first-order derivative present in the operator $\mathbb{T}$ would contaminate the constant kernel $\mathbb{K}$ in view of the integration by part formula:

$$
\int \boldsymbol{d} \boldsymbol{r} \mathrm{e}^{-\mathrm{i} \boldsymbol{Q}_{\boldsymbol{H}} \cdot \boldsymbol{r}} \mathrm{e}^{-\mathrm{i} \mathrm{Q}_{z} \eta} \nabla \eta=-\frac{\boldsymbol{Q}_{H}}{Q_{z}} \int \boldsymbol{d} \boldsymbol{r} \mathrm{e}^{-\mathrm{i} \boldsymbol{Q}_{\boldsymbol{H}} \cdot \boldsymbol{r}} \mathrm{e}^{-\mathrm{i} \mathrm{Q}_{z} \eta},
$$

Hence a parabolic correction to the tangent plane approximation can only involve second-order derivatives of the surface. This is why it is legitimate to call this additional term a "curvature correction" to the surface. Now, curvature components in a Kirchhoff integral bring in quadratic corrections in slope, as can be seen from the integration by part formula:

$$
\int(\nabla \eta)^{2} \mathrm{e}^{-\mathrm{i} \boldsymbol{Q}_{\boldsymbol{H}} \cdot \boldsymbol{r}} \mathrm{e}^{-\mathrm{i} \mathrm{Q}_{\mathrm{z}} \eta} \boldsymbol{d} \boldsymbol{r}=\frac{Q_{H}^{2}}{Q_{z}^{2}} \int \mathrm{e}^{-\mathrm{i} \boldsymbol{Q}_{\boldsymbol{H}} \cdot \boldsymbol{r}} \mathrm{e}^{-\mathrm{i} \mathrm{Q}_{\mathrm{z}} \eta} \boldsymbol{d} \boldsymbol{r}-\frac{\mathrm{i}}{Q_{z}} \int \Delta \eta \mathrm{e}^{-\mathrm{i} \boldsymbol{Q}_{\boldsymbol{H}} \cdot \boldsymbol{r}} \mathrm{e}^{-\mathrm{i} Q_{z} \eta} \boldsymbol{d} \boldsymbol{r}
$$

or more generally

$$
\begin{aligned}
\int \mathbb{T}(\boldsymbol{\nabla} \eta) \mathrm{e}^{-\mathrm{i} \boldsymbol{Q}_{\boldsymbol{H}} \cdot \boldsymbol{r}} \mathrm{e}^{-\mathrm{i} \mathrm{Q}_{\mathrm{z}} \eta} \boldsymbol{d} \boldsymbol{r} & =\mathbb{T}\left(-\frac{\boldsymbol{Q}_{H}}{Q_{z}}\right) \int \mathrm{e}^{-\mathrm{i} \boldsymbol{Q}_{\boldsymbol{H}} \cdot \boldsymbol{r}} \mathrm{e}^{-\mathrm{i} \mathrm{Q}_{\mathrm{z}} \eta} \boldsymbol{d} \boldsymbol{r} \\
& -\frac{\mathrm{i}}{Q_{z}} \int \mathbb{T}(\boldsymbol{\nabla})[\eta] \mathrm{e}^{-\mathrm{i} \boldsymbol{Q}_{\boldsymbol{H}} \cdot \boldsymbol{r}} \mathrm{e}^{-\mathrm{i} \mathrm{Q}_{\mathrm{z}} \eta} \boldsymbol{d} \boldsymbol{r}
\end{aligned}
$$

for any second-order differential operator $\mathbb{T}$. Here the tensor $\mathbb{T}(\boldsymbol{\nabla} \eta)$ is the polynomial of slopes obtained by formal replacement of order of derivatives by order in slopes in the defining equation (2.4) $\left(\partial_{x}^{n} \partial_{y}^{m} \eta \rightarrow\left(\partial_{x} \eta\right)^{n}\left(\partial_{y} \eta\right)^{m}\right)$ and $\mathbb{T}\left(-\frac{\boldsymbol{Q}_{H}}{Q_{z}}\right)$ is the same polynomial evaluated at the stationary slope $\nabla \eta=-\boldsymbol{Q}_{H} / Q_{z}$. The operator $\mathbb{T}(\boldsymbol{\nabla})$ will be referred to as the "curvature kernel". A generalization of the integration by part formula (2.6) to higher-order derivatives would show that any derivative of order $N$ in a Kirchhoff integral can be transformed into a polynomial function of slope of degree $N$. This elucidates the role of the higherorder derivatives in the functional (2.4) and shows that their omission results in an cubic error in slope.

The curvature correction (2.3) can therefore be rewritten in the form:

$$
\mathbb{S}=\frac{1}{Q_{z}} \int \frac{\boldsymbol{d} \boldsymbol{r}}{(2 \pi)^{2}}\left(\mathbb{K}+\mathbb{T}\left(-\frac{\boldsymbol{Q}_{H}}{Q_{z}}\right)-\mathbb{T}(\boldsymbol{\nabla} \eta)\right) \mathrm{e}^{-\mathrm{i} \boldsymbol{Q}_{\boldsymbol{H}} \cdot \boldsymbol{r}} \mathrm{e}^{-\mathrm{i} Q_{z} \eta},
$$

By construction, the above scattering amplitude is consistent with the KA. Now, a unified model should also be consistent with the SPM scattering amplitude in the limit of small elevations:

$$
\mathbb{S}=-\mathrm{i} \mathbb{B} \widehat{\eta}\left(\boldsymbol{Q}_{\mathrm{H}}\right),
$$


where $\widehat{\eta}$ is the Fourier transform of the surface:

$$
\widehat{\eta}\left(\boldsymbol{Q}_{H}\right)=\int \frac{d \boldsymbol{r}}{(2 \pi)^{2}} \mathrm{e}^{-\mathrm{i} \boldsymbol{Q}_{H} \cdot \boldsymbol{r}} \eta(\boldsymbol{r}),
$$

and $\mathbb{B}$ is the so-called Bragg tensor, which can also be found in the appendix of [1]. Assuming $Q_{z} \eta<<1$ in the integral (2.9) and linearizing the exponential, we see that the consistency with the SPM limit sets the constraint:

$$
\mathbb{T}\left(-\frac{Q_{H}}{Q_{z}}\right)=(\mathbb{B}-\mathbb{K})
$$

The identification of the right hand side with a curvature kernel is possible because the difference of the kernels $\mathbb{B}$ and $\mathbb{K}$ is quadratic in $Q_{H}$, as was shown in [8]. Actually, this difference is quadratic in its lowest-order terms only, as higherorder terms enter in the expression. Only in the particular case of conducting surfaces in backscattering configuration is it exactly quadratic. The curvature correction (2.9) is now structurally identical to the WCA, where the scattering amplitude was postulated in the form:

$$
\mathbb{S}=\frac{1}{Q_{z}} \int \frac{\boldsymbol{d} \boldsymbol{r}}{(2 \pi)^{2}}(\mathbb{B}-\mathbb{T}(\boldsymbol{\nabla} \eta)) \mathrm{e}^{-\mathrm{i} \boldsymbol{Q}_{H} \cdot \boldsymbol{r}} \mathrm{e}^{-\mathrm{i} Q_{z} \eta}
$$

with a nonlinear kernel of at least quadratic order in slope, satisfying the requirement (2.12). We will therefore refer to any scattering amplitude of the form (2.13) as a "Weighted Curvature Approximation", as long as the involved kernel is approximatively or exactly quadratic in slope.

For the two-dimensional problem where the surface depends on one single space variable, say $\eta(x)$, the identity (2.12) suffices to obtain the quadratic kernel $T\left(\eta^{\prime}\right)$ (a scalar in that case):

$$
T\left(\eta^{\prime}\right)=(B-K) \frac{Q_{z}^{2}}{Q_{H}^{2}} \eta^{\prime 2}
$$

For the full three dimensional problem, equation (2.12) is not enough to completely determine the quadratic kernel, due to the multiplicity of admissible kernels. For example,

$$
\mathbb{T}(\boldsymbol{\nabla} \eta)=(\mathbb{B}-\mathbb{K}) \frac{Q_{z}^{2}}{Q_{H}^{2}}(\nabla \eta)^{2} \Leftrightarrow \mathbb{T}(\boldsymbol{\nabla})[\eta]=(\mathbb{B}-\mathbb{K}) \frac{Q_{z}^{2}}{Q_{H}^{2}} \Delta \eta,
$$

or

$\mathbb{T}(\boldsymbol{\nabla} \eta)=(\mathbb{B}-\mathbb{K}) \frac{Q_{z}^{4}}{Q_{H}^{4}}\left(\frac{\boldsymbol{Q}_{H}}{Q_{z}} \cdot \nabla \eta\right)^{2} \Leftrightarrow \mathbb{T}(\boldsymbol{\nabla})[\eta]=(\mathbb{B}-\mathbb{K}) \frac{Q_{z}^{4}}{Q_{H}^{4}}\left(\frac{\boldsymbol{Q}_{H}}{Q_{z}} \cdot \nabla \nabla \eta \cdot \frac{\boldsymbol{Q}_{H}}{Q_{z}}\right)$

are two admissible instances of $\mathbb{T}$ in view of equation (2.12). However, we will see that this ambiguity can be removed for sea surfaces (section 4). 


\section{The link with the original Weighted Curva- ture Approximation}

In the original WCA $[8,9]$, the kernel was designed heuristically in order to satisfy the required limiting properties, namely the SPM and KA limits, together with the tilt invariance at first-order in slope. It was similar to the previous form (2.15) and (2.16) inasmuch as it involved the difference of a Bragg and Kirchhoff kernel, but the quadratic dependence in the slope variable was reached by substituting the latter to the $\boldsymbol{Q}_{H}$ variable in the expressions of the dyads:

$$
\mathbb{T}_{W C A}(\nabla \eta)=(\mathbb{B}-\mathbb{K})_{Q_{H} \rightarrow-Q_{z} \nabla \eta}
$$

The advantage of this technique is to relax the constraint that the kernel be exactly quadratic, thus allowing the occurrence of high-order terms in slope. However, the resulting expression is more complex and less practical when it comes to statistical averaging. To ease the comparison with the quadratic kernels (2.15) or (2.16), let us focus on the conducting case. The Bragg dyad in the perfectly conducting case is given by $([3,1]$ :

$\mathbb{B}=\frac{1}{2}(\boldsymbol{W}+\boldsymbol{Q}) \cdot \hat{z} \hat{z} \cdot(\boldsymbol{W}-\boldsymbol{Q}) \hat{\mathbf{1}} \hat{\mathbf{1}}+\hat{z} \hat{z} \cdot(Q Q-\boldsymbol{W} \boldsymbol{W})+(Q Q-\boldsymbol{W} \boldsymbol{W}) \cdot \hat{z} \hat{z}-Q^{2} \hat{z} \hat{z}$

where $\boldsymbol{W}=\boldsymbol{K}+\boldsymbol{K}_{0}$ and $\boldsymbol{Q}=\boldsymbol{K}-\boldsymbol{K}_{0}$. The Kirchhoff dyad in the perfectly conducting case is given by:

$$
\mathbb{K}=-\frac{1}{2} Q^{2} \hat{\mathbf{1}} \hat{\mathbf{1}}+Q Q
$$

The Kirchhoff dyad is obtained from the Bragg dyad by operating the formal replacement $\hat{z} \rightarrow \hat{\boldsymbol{Q}}$. Considering for simplicity the backscattering direction only $(\boldsymbol{W}=\mathbf{0}) \mathrm{n}$, we obtain the following expression for the difference of the Bragg and Kirchhoff dyad:

$$
\mathbb{B}-\mathbb{K}=\frac{1}{2} Q_{H}^{2} \hat{\mathbf{1}} \hat{\mathbf{1}}-\boldsymbol{Q}_{H} \boldsymbol{Q}_{H}-Q_{H}^{2} \hat{\boldsymbol{z}} \hat{\boldsymbol{z}}
$$

Now operating the replacement

$$
\left(\boldsymbol{Q}_{H}, Q_{z}, \boldsymbol{Q}\right) \rightarrow\left(-Q_{z} \boldsymbol{\nabla} \eta, \sqrt{Q^{2}-\left(Q_{z} \nabla \eta\right)^{2}},-Q_{z} \boldsymbol{\nabla} \eta+\sqrt{Q^{2}-\left(Q_{z} \nabla \eta\right)^{2}} \hat{\boldsymbol{z}}\right),
$$

we obtain a kernel which is actually quadratic:

$$
\mathbb{T}_{W C A}(\boldsymbol{\nabla} \eta)=\left(\frac{1}{2}(\nabla \eta)^{2} \hat{\mathbf{1}} \hat{\mathbf{1}}-\nabla \eta \nabla \eta-(\nabla \eta)^{2} \hat{z} \hat{z}\right) Q_{z}^{2}
$$

but not as simple as the Laplacian kernel (2.15). Note that

$$
\mathbb{T}_{W C A}\left(-\frac{\boldsymbol{Q}_{H}}{Q_{z}}\right)=\frac{1}{2} Q_{H}^{2} \hat{\mathbf{1}} \hat{\mathbf{1}}-\boldsymbol{Q}_{H} \boldsymbol{Q}_{H}-Q_{H}^{2} \hat{\boldsymbol{z}} \hat{\boldsymbol{z}}=\mathbb{B}-\mathbb{K}
$$


so that the usual requirement $(2.12)$ is satisfied. In the dielectric case, the dyadic Kirchhoff and Bragg kernels involve the Fresnel reflection coefficients at the local angles, which are fully nonlinear function of the slope. Hence, the WCA kernel in that case is only approximatively quadratic and must be computed numerically.

\section{The universal Weighted Curvature Approxi- mation for sea spectra}

The previous study has shown that the kernel of the WCA for the full threedimensional problem is only ambiguously defined and cannot be determined by the sole constraint on the asymptotic low- and high-frequency regime. However, under the assumption of Gaussian statistics, we will now show that all admissible kernels lead to the same expression for the off-specular incoherent NRCS in the case of a sea surface. Consider a scattering amplitude satisfying the requirement (2.3) of a weighted curvature approximation, with a quadratic kernel $\mathbb{T}$ satisfying eq. (2.12). Consider a Gaussian stationary surface with correlation function $\rho$ and power spectrum:

$$
\Gamma(\boldsymbol{k})=\frac{1}{(2 \pi)^{2}} \int \boldsymbol{d} \boldsymbol{r} \mathrm{e}^{-\mathrm{i} \boldsymbol{k} \cdot \boldsymbol{r}} \rho(\boldsymbol{r})
$$

From the statistical formulas in Appendix 8.1 the incoherent field is found to be:

$$
\sigma=\sigma^{(11)}+\sigma^{(12)}+\sigma^{(22)}
$$

where the first term is the KA NRCS,

$$
\sigma^{(11)}=\frac{1}{Q_{z}^{2}}|\mathbb{K}|^{2} \int \frac{\boldsymbol{d} \boldsymbol{r}}{\pi} \mathrm{e}^{-\mathrm{i} \boldsymbol{Q}_{\boldsymbol{H}} \cdot \boldsymbol{r}}\left[\mathrm{e}^{-Q_{z}^{2}\left(\rho_{0}-\rho\right)}-\mathrm{e}^{-Q_{z}^{2} \rho_{0}}\right]
$$

and the remaining term are the contribution of the curvature correction:

$$
\begin{aligned}
\sigma^{(12)} & =\frac{1}{Q_{z}^{2}} 2 \Re\left[\mathbb { K } ^ { * } \int \frac { \boldsymbol { d } \boldsymbol { r } } { \pi } \mathrm { e } ^ { - \mathrm { i } \boldsymbol { Q } _ { \boldsymbol { H } } \cdot \boldsymbol { r } } \left[\mathrm{e}^{-Q_{z}^{2}\left(\rho_{0}-\rho\right)}\left\{\mathbb{T}(\boldsymbol{\nabla})[\rho]-(\mathbb{T}(\boldsymbol{\nabla})[\rho])_{0}\right\}\right.\right. \\
& \left.\left.\left.-\mathrm{e}^{-Q_{z}^{2} \rho_{0}} \mathbb{T}(\boldsymbol{\nabla})[\rho]\right)_{0}\right]\right] \\
\sigma^{(22)} & =\frac{1}{Q_{z}^{2}} \int \frac{\boldsymbol{d} \boldsymbol{r}}{\pi} \mathrm{e}^{-\mathrm{i} \boldsymbol{Q}_{\boldsymbol{H}} \cdot \boldsymbol{r}}\left[\mathrm{e}^{-Q_{z}^{2}\left(\rho_{0}-\rho\right)}\left\{\mathbb{T}^{2}(\boldsymbol{\nabla})\right)[\rho]-Q_{z}^{2}\left|\mathbb{T}(\boldsymbol{\nabla})[\rho]-(\mathbb{T}(\boldsymbol{\nabla})[\rho])_{0}\right|^{2}\right\} \\
& \left.+Q_{z}^{2} \mathrm{e}^{-Q_{z}^{2} \rho_{0}}\left(\mathbb{T}^{2}(\boldsymbol{\nabla})[\rho]\right)_{0}\right]
\end{aligned}
$$

In the last equation, the term $\mathbb{T}^{2}(\nabla)$ indicates two successive applications of the differential operator (for example $\mathbb{T}^{2}(\nabla) \rho=\mathbb{T}_{0}^{2} \Delta \Delta \rho$ whenever $\mathbb{T}(\boldsymbol{\nabla}) \rho=$ $\left.\mathbb{T}_{0} \Delta \rho\right)$. Here and everywhere, the product or ratio of two dyads is to be understood in the sense of the corresponding matrix-element operation. 
It is interesting to note that the corrective terms $\sigma^{(12)}$ and $\sigma^{(22)}$ vanish in the case of a quadratic correlation function $\left(\rho(\boldsymbol{r})=\rho_{0}+\boldsymbol{r} \cdot \mathbb{A} \boldsymbol{r}\right)$, making the present NRCS a parabolic rather than tangent plane approximation. For mono-scale surfaces where the correlation function is well approximated by a parabolic behavior at small lags, the corrective terms are thus expected to be small. Hence, the supplementary terms in the NRCS can be seen as diffraction correction linked to the multi-scale nature of the surface.

In a good approximation, these integrals do not depend on the specific form of the kernel, as long as (2.12) is satisfied, due to the filtering effect of the integrand around the Bragg frequency. This approximation, termed the "Kirchhoff filtering formula", is proved in the Appendix 8.2 and allows for considerable simplification at off-specular angles :

$$
\frac{1}{(2 \pi)^{2}} \int \boldsymbol{d} \boldsymbol{r} \mathrm{e}^{-\mathrm{i} \boldsymbol{Q}_{\boldsymbol{H}} \cdot \boldsymbol{r}} \mathrm{e}^{-Q_{z}^{2}\left(\rho_{0}-\rho\right)} \mathbb{T}^{n}(\boldsymbol{\nabla})[\rho] \simeq \mathbb{T}^{n}\left(-\frac{\boldsymbol{Q}_{H}}{Q_{z}}\right) \Gamma\left(\boldsymbol{Q}_{H}\right),
$$

where $n=1,2$. In $\sigma^{(22)}$ the contribution $Q_{z}^{2}\left[\mathbb{T}(\boldsymbol{\nabla})[\rho]-(\mathbb{T}(\boldsymbol{\nabla})[\rho])_{0}\right]^{2}$ is of fourth order in slope and can be neglected. This leads to the following formula for the off-specular incoherent NRCS:

$$
\begin{aligned}
\sigma & =\frac{|\mathbb{K}|^{2}}{Q_{z}^{2}}\left(1+2 \Re\left[\frac{(\mathbb{T}(\boldsymbol{\nabla})[\rho])_{0}}{\mathbb{K}^{*}}\right]\right) \int \frac{\boldsymbol{d} \boldsymbol{r}}{\pi} \mathrm{e}^{-\mathrm{i} \boldsymbol{Q}_{\boldsymbol{H}} \cdot \boldsymbol{r}}\left[\mathrm{e}^{-Q_{z}^{2}\left(\rho_{0}-\rho\right)}-\mathrm{e}^{-Q_{z}^{2} \rho_{0}}\right] \\
& -\frac{|\mathbb{K}|^{2}}{Q_{z}^{2}} 2 \Re\left(\int \boldsymbol{d} \boldsymbol{r} \mathrm{e}^{-\mathrm{i} \boldsymbol{Q}_{\boldsymbol{H}} \cdot \boldsymbol{r}} \mathrm{e}^{-Q_{z}^{2} \rho_{0}}\left[\frac{(\mathbb{T}(\boldsymbol{\nabla})[\rho])}{\mathbb{K}^{*}}\right]\right)+\left[2 \Re\left[(\mathbb{B}-\mathbb{K}) \mathbb{K}^{*}\right]+|\mathbb{B}-\mathbb{K}|^{2}\right] 4 \pi \Gamma\left(\boldsymbol{Q}_{H}\right)
\end{aligned}
$$

Now

$$
\left|\frac{\mathbb{T}(\boldsymbol{\nabla})[\rho]}{\mathbb{K}^{*}}\right| \simeq\left|\frac{\mathbb{B}-\mathbb{K}}{\mathbb{K}}\right| \times \text { m.s.s, }
$$

where both the ratio of the kernels and the mean square slope (m.s.s) are small, so that the two terms in the integrand involving this ratio can also be neglected. This makes it possible to recast the last expression in a form which highlights the difference with the Bragg limit:

$$
\sigma=\left|\frac{\mathbb{K}}{Q_{z}}\right|^{2}\left(\int \frac{\boldsymbol{d} \boldsymbol{r}}{\pi} \mathrm{e}^{-\mathrm{i} \boldsymbol{Q}_{\boldsymbol{H}} \cdot \boldsymbol{r}} \mathrm{e}^{-Q_{z}^{2}\left(\rho_{0}-\rho\right)}-4 \pi Q_{z}^{2} \Gamma\left(\boldsymbol{Q}_{H}\right)\right)+|\mathbb{B}|^{2} 4 \pi \Gamma\left(\boldsymbol{Q}_{H}\right)
$$

We will refer to this surprisingly simple formula, which is the central result of this paper, merely as the Weighted Curvature Approximation for sea spectra, as no ambiguity remains. This expression of the incoherent NRCS is a priori devoted to broad band spectra. However, the consistency with the fundamental Bragg and Kirchhoff regime can easily be inspected. The SPM incoherent NRCS $\left(|\mathbb{B}|^{2} 4 \pi \Gamma\left(\boldsymbol{Q}_{H}\right)\right)$ is clearly recovered in the small-scale limit $Q_{z}^{2} \rho_{0} \rightarrow 0$. The KA limit is also obtained by setting the Bragg component to zero $\left(\Gamma\left(\boldsymbol{Q}_{H}\right)=0\right)$, which amounts to kill the small-scale components. Note that the formula (4.32) does not predict any cross-polarization in the incidence plane, as neither does the Kirchhoff nor the Bragg kernel. 


\section{The link with the Resonant Curvature Ap- proximation}

The Resonant Curvature Approximation is a specific instance of a more general method, called the Local Curvature Approximation [8]. The scattering amplitude is taken in the same functional form as the second-order small-slope approximation of Voronovich [12], but is written as a correction of the Kirchhoff scattering amplitude:

$$
\mathbb{S}=\frac{\mathbb{K}}{Q_{z}} \int \frac{\boldsymbol{d} \boldsymbol{r}}{(2 \pi)^{2}} \mathrm{e}^{-\mathrm{i} \boldsymbol{Q}_{\boldsymbol{H}} \cdot \boldsymbol{r}} \mathrm{e}^{-\mathrm{i} \mathrm{Q}_{z} \eta}-\mathrm{i} \int \boldsymbol{d} \boldsymbol{d} \boldsymbol{\xi} \mathbb{M}(\boldsymbol{\xi}) \widehat{\eta}(\boldsymbol{\xi}) \mathrm{e}^{-\mathrm{i} Q_{z} \eta} \mathrm{e}^{-\mathrm{i}\left(\boldsymbol{Q}_{\boldsymbol{H}}-\boldsymbol{\xi}\right) \cdot \boldsymbol{r}}
$$

where $\mathbb{M}(\boldsymbol{\xi})$ is a kernel which varies from one method to the other. Interestingly, the second term on the right-hand side of (5.33) reduces to the form (2.3) whenever $\mathbb{M}(\boldsymbol{\xi})$ is a polynomial function of $\boldsymbol{\xi}$, in which case the Fourier integral over this variable becomes a local differential operator. However, the form given in (5.33) is more general than (2.3) as it permits nonlocal corrections for other types of tensors $\mathbb{M}$ (see for instance the discussion in paragraph 6.1 of [1]). Standard manipulations on stationary Gaussian processes lead to the following expression for the cross-term in the incoherent NRCS (the leading term $\sigma^{(11)}$ is unchanged):

$\sigma^{(12)} \simeq 2 \Re\left[\mathbb{K} \int \frac{\boldsymbol{d} \boldsymbol{r}}{\pi} \mathrm{e}^{-\mathrm{i} \boldsymbol{Q}_{\boldsymbol{H}} \cdot \boldsymbol{r}}\left[\mathrm{e}^{-Q_{z}^{2}\left(\rho_{0}-\rho\right)}-\mathrm{e}^{-Q_{z}^{2} \rho_{0}}\right] \int \boldsymbol{d} \boldsymbol{\xi} \mathbb{M}^{*}(\boldsymbol{\xi}) \Gamma(\boldsymbol{\xi})\left(\mathrm{e}^{\mathrm{i} \boldsymbol{\xi} \cdot \boldsymbol{r}}-1\right)\right]$

The term $\sigma^{(22)}$ is neglected for simplicity. This is justified whenever the relative correction to the KA is small in the scattering amplitude, a condition which is in general not granted and must be verified a posteriori.

In the RCA, the kernel $\mathbb{M}(\boldsymbol{\xi})$ is taken as:

$$
\mathbb{M}(\boldsymbol{\xi})=(\mathbb{B}-\mathbb{K}) \phi(\boldsymbol{\xi})
$$

where is $\phi$ a tight real filter around the Bragg frequency (actually a Dirac function in its original formulation, which we here extend to nonsingular filters for mathematical properness and numerical purposes). The NRCS correction can therefore be simplified to:

$$
\sigma^{(12)} \simeq 2 \Re\left[\frac{\mathbb{K}\left(\mathbb{B}^{*}-\mathbb{K}^{*}\right)}{Q_{H}^{2}} \int \frac{\boldsymbol{d} \boldsymbol{r}}{\pi} \mathrm{e}^{-\mathrm{i} \boldsymbol{Q}_{H} \cdot \boldsymbol{r}} \mathrm{e}^{-Q_{z}^{2}\left(\rho_{0}-\rho\right)}\left[\left(\Delta \rho_{f}\right)_{0}-\Delta \rho_{f}(\boldsymbol{r})\right]\right],
$$

where $\rho_{f}$ and $\Gamma_{f}$ are filtered correlation function and spectrum, respectively:

$$
\rho_{f}(\boldsymbol{r})=\int \boldsymbol{d} \boldsymbol{\xi} \Gamma_{f}(\boldsymbol{\xi}) \mathrm{e}^{\mathrm{i} \boldsymbol{\xi} \cdot \boldsymbol{r}}, \Gamma_{f}(\boldsymbol{\xi})=\frac{Q_{H}^{2}}{\xi^{2}} \phi(\boldsymbol{\xi}) \Gamma(\boldsymbol{\xi})
$$

Note that $\phi$ must enjoy the same properties as a true power spectrum, and therefore be positive and centro-symmetric. The RCA term can thus be rewritten in 
the form of a curvature correction:

$$
\sigma^{(12)} \simeq \frac{2}{Q_{z}^{2}} \Re\left[\mathbb{K} \int \frac{\boldsymbol{d} \boldsymbol{r}}{\pi} \mathrm{e}^{-\mathrm{i} \boldsymbol{Q}_{H} \cdot \boldsymbol{r}} \mathrm{e}^{-Q_{z}^{2}\left(\rho_{0}-\rho\right)}\left(T(\boldsymbol{\nabla})\left[\rho_{f}\right]-\left(T(\boldsymbol{\nabla})\left[\rho_{f}\right]\right)_{0}\right)\right]
$$

with the following choice for the kernel $\mathbb{T}$ :

$$
\mathbb{T}(\nabla)[\rho]=\frac{Q_{z}^{2}}{Q_{H}^{2}}(\mathbb{B}-\mathbb{K}) \Delta \rho_{f}
$$

Now the Kirchhoff filtering formula also applies in this case,

$$
\frac{1}{(2 \pi)^{2}} \int \boldsymbol{d} \boldsymbol{r} \mathrm{e}^{-\mathrm{i} \boldsymbol{Q}_{H} \cdot \boldsymbol{r}} \mathrm{e}^{-Q_{z}^{2}\left(\rho_{0}-\rho\right)} \mathbb{T}(\boldsymbol{\nabla})\left[\rho_{f}\right] \simeq \mathbb{T}\left(-\frac{\boldsymbol{Q}_{H}}{Q_{z}}\right) \Gamma_{f}\left(\boldsymbol{Q}_{H}\right)
$$

leading to

$$
\sigma^{(12)} \simeq 2 \Re\left[\mathbb{K}^{*}(\mathbb{B}-\mathbb{K})\right] \phi\left(\boldsymbol{Q}_{H}\right) 4 \pi \Gamma\left(\boldsymbol{Q}_{H}\right),
$$

which is the cross-term of the universal WCA (4.32) if the filter meets the condition $\phi\left(\boldsymbol{Q}_{H}\right)=1$.

In the original RCA papers [5, 6], the calculation of the total cross-section $\sigma^{(11)}+\sigma^{(12)}$ was realized by considering the corrective term $\sigma^{(12)}$ as a phase perturbation of the leading Kirchhoff NRCS $\sigma^{(11)}$. This astute manipulation was first introduced in [13] for the numerical evaluation of the second-order SmallSlope Approximation and is often invoked to simplify the otherwise difficult computations. With this phase approximation, the calculation of the RCA cross-section is equivalent to a Kirchhoff NRCS for an effective surface with modified, polarization dependent, power spectrum:

$$
\tilde{\Gamma}(\boldsymbol{\xi})=\left|1+\frac{(\mathbb{B}-\mathbb{K})}{\mathbb{K}} \phi\left(\boldsymbol{\xi}-\boldsymbol{Q}_{H}\right)\right|^{2} \Gamma(\boldsymbol{\xi})
$$

In the numerical routines the filter $\phi$ is usually realized through a tight Gaussian around the Bragg vector, together with its centrosymmetric version:

$$
\phi(\boldsymbol{\xi})=\exp \left(-\alpha\left\|\xi-\boldsymbol{Q}_{H}\right\|^{2}\right)+\exp \left(-\alpha\left\|\xi+\boldsymbol{Q}_{H}\right\|^{2}\right)
$$

This necessitates the introduction of a somewhat arbitrary parameter $\alpha$ which controls the width of the filter and can be used to give more or less weight to the Bragg frequency. Note that extreme variations of $\alpha$ brings the modified spectrum $\tilde{\Gamma}$ either close to the actual spectrum $\Gamma$ or to its dilated version $\left|1+\frac{(\mathbb{B}-\mathbb{K})}{\mathbb{K}}\right|^{2} \Gamma$. Efficient values of the filter are those which modifies the spectrum over a few electromagnetic wavenumbers around the Bragg frequency. Figure 1 shows an example of RCA computation in $\mathrm{C}$ band for an isotropic Elfouhaily spectrum at $10 \mathrm{~m} / \mathrm{s}$ wind speed, for different values of the filter parameter $\left(\alpha=410^{-4}\right.$ and $\left.\alpha=1610^{-2}\right)$. As can be seen on the plot, the resulting NRCS is drastically dependent upon the choice of this parameter, especially in $\mathrm{HH}$ polarization. This is the main limitation of the method. 


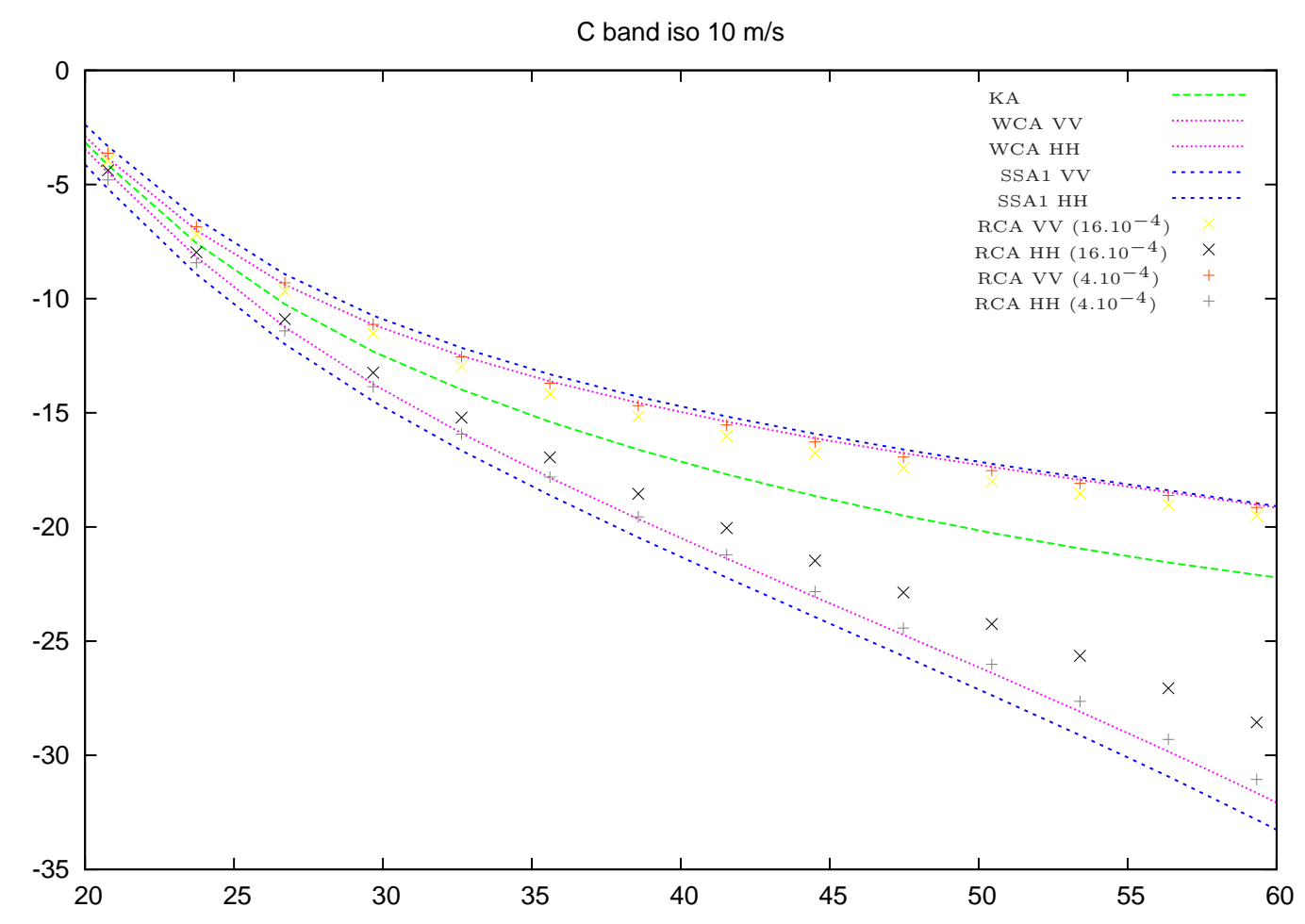

Figure 1: C-Band backscattering NRCS for an isotropic Elfouhaily spectrum at $10 \mathrm{~m} / \mathrm{s}$ wind speed. The RCA co-polarizations are shown for different values of the filter $\alpha=4 \cdot 10^{-4}$ and $\alpha=16 \cdot 10^{-4}$. KA, WCA and SSA1 results are given for comparison.

\section{Some numerical results}

To evaluate the WCA in its universal form (4.32), we will perform some numerical comparisons with the Method of Moments (MoM) in some test cases where this is possible. Today, MoM computations in the microwave regime for fully two-dimensional and dielectric sea surfaces by even moderate wind is still a challenging issue, unless large scales are truncated. We have therefore devised some simplified configurations which make it possible to deal with a complete spectrum:

1. 1D perfectly conducting surfaces with Elfouhaily omnidirectional spectrum, by a $10 \mathrm{~m} / \mathrm{s}$ wind speed in $\mathrm{L}$ band, in the monostatic case.

2. 2D perfectly conducting surfaces with Elfouhaily directional spectrum, by a $7 \mathrm{~m} / \mathrm{s}$ wind speed in L band. Only the bistatic configuration in the plane of incidence has been considered, since it is numerically less demanding. 
In addition we have performed some comparison with the cut-off invariant TwoScale Model (GO-SSA) [11] at different wind speed and frequency bands.

\subsection{Comparison with 1D MoM}

We consider the scattering problem at $25 \mathrm{~cm}$ (L-band) electromagnetic wavelength in vacuum, for a 1D perfectly conducting rough surface with Elfouhaily omnidirectional spectrum at $10 \mathrm{~m} / \mathrm{s}$ wind speed . The incident field is a Gaussian beam with $100 \mathrm{~m}$ standard deviation, which is of the order of the ocean peak wave length. The discretization step is one eighth of the wavelength and length of the samples profiles is taken to 5 standard deviations. The central incidence of beam runs from nadir to $75^{\circ}$ with a $5^{\circ}$ step. 1D perfectly conducting integral equations for both polarization cases are discretized with pulse expansion functions and delta testing functions. The resulting linear system is solved iteratively with the GMRES algorithm, for each incidence. The matrixvector product is performed via the Sparse Matrix Canonical Grid scheme [14]. Strong interaction length is set to 30 wavelengths, and farther interactions are interpolated over a 2D grid with 32 vertical levels. A Monte Carlo average is performed over 100 samples.

Figure 2 display the monostatic NRCS versus the incidence angle for MoM, KA and WCA from the simplified formula (4.32). A comparison is also given with the first-order Small-Slope Approximation (SSA1) of Voronovich [12], which is one of the other most frequently used methods in scattering from sea surface. We recall that SSA1 is obtained through the KA NRCS (4.26) with the replacement of the Kirchhoff kernel $\mathbb{K}$ by the Bragg kernel $\mathbb{B}$. As expected, all methods coincide around nadir, where backscattering shows no difference between polarizations. At higher incidence angles, KA becomes less accurate, being polarization insensitive. On the contrary, SSA1 overestimates the polarization effect: it strongly underestimate the horizontal polarization and slightly underestimate the vertical polarization. WCA remains extremely accurate over the whole scattering diagram.

At large angles, the MoM backscattering cross-section does not decrease as expected but reaches a plateau. Careful sampling convergence tests and an estimation of the error through the extinction theorem and an energy balance have been realized. An explanation for the small differences of the MOM and analytical theories in this region is still being sought.

\subsection{Comparison with 2D MoM}

For two-dimensional surfaces, the Small Slope Integral Equation [15] is applied. The strong interaction distance is one half wavelength, and height differences are neglected in the distance computations for farther interactions. At L-band by a $7 \mathrm{~m} / \mathrm{s}$ wind speed and a $44 \mathrm{~m}$ peak wavelength, the beam width is set to $34 \mathrm{~m}$. The sampling rate of the surface is one eighth of the EM wavelength in the air. The incoherent NRCS is obtained through a Monte Carlo average. The surface 
$\mathrm{L}$ band, $10 \mathrm{~m} / \mathrm{s}$, monostatic NRCS

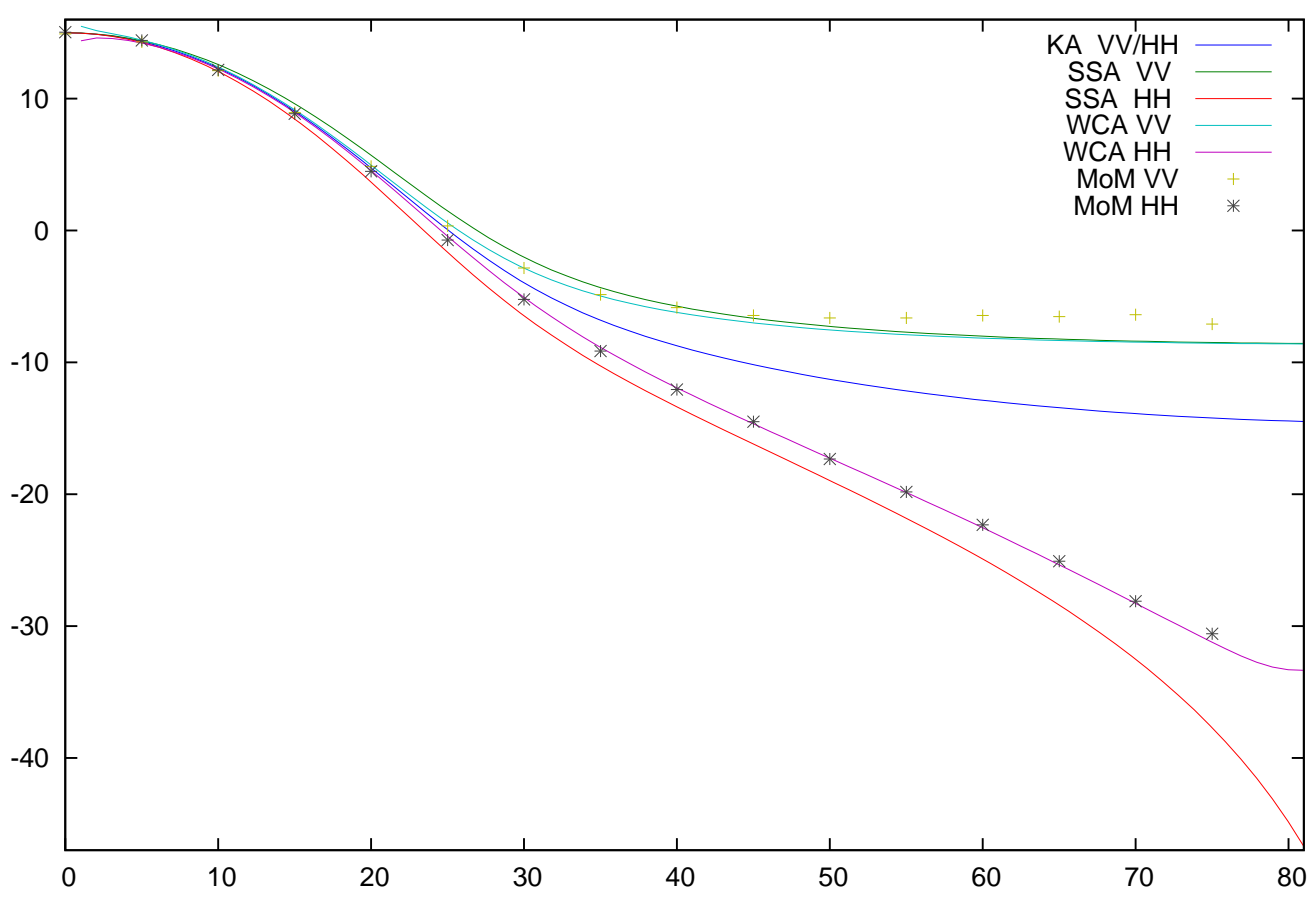

Figure 2: 1D perfectly conducting surfaces, Omnidirectional Elfouhaily spectrum, $10 \mathrm{~m} / \mathrm{s}$, L band. Comparison of KA, SSA1 and WCA with MoM. Monostatic NRCS.

is assumed Gaussian with a fully developed directional Elfouhaily spectrum. The incidence angle is taken to 40 degrees, with an upwind illumination.

Figure 3 displays the bistatic NRCS for KA, SSA WCA and MoM in the plane of incidence. A blow-up at moderate angles is shown on Figure 4. The still visible oscillations of the MoM are due to an insufficient number of sample surfaces, a drastic restriction imposed by the large size of the latter and the consequently prohibitive computational time. The MoM values at very large angles (above 80 degrees incidence, not represented here) are not reliable since they reach the limit of the numerical dynamics. The same conclusions can be drawn as in the $1 \mathrm{D}$ monostatic case for the off-specular region. Note the unphysical behavior of the WCA NRCS around the specular region, due to the low-frequency divergence of the involved spectrum at Bragg frequency, and recall that the approximation (4.32) of WCA holds only away from the specular region. 


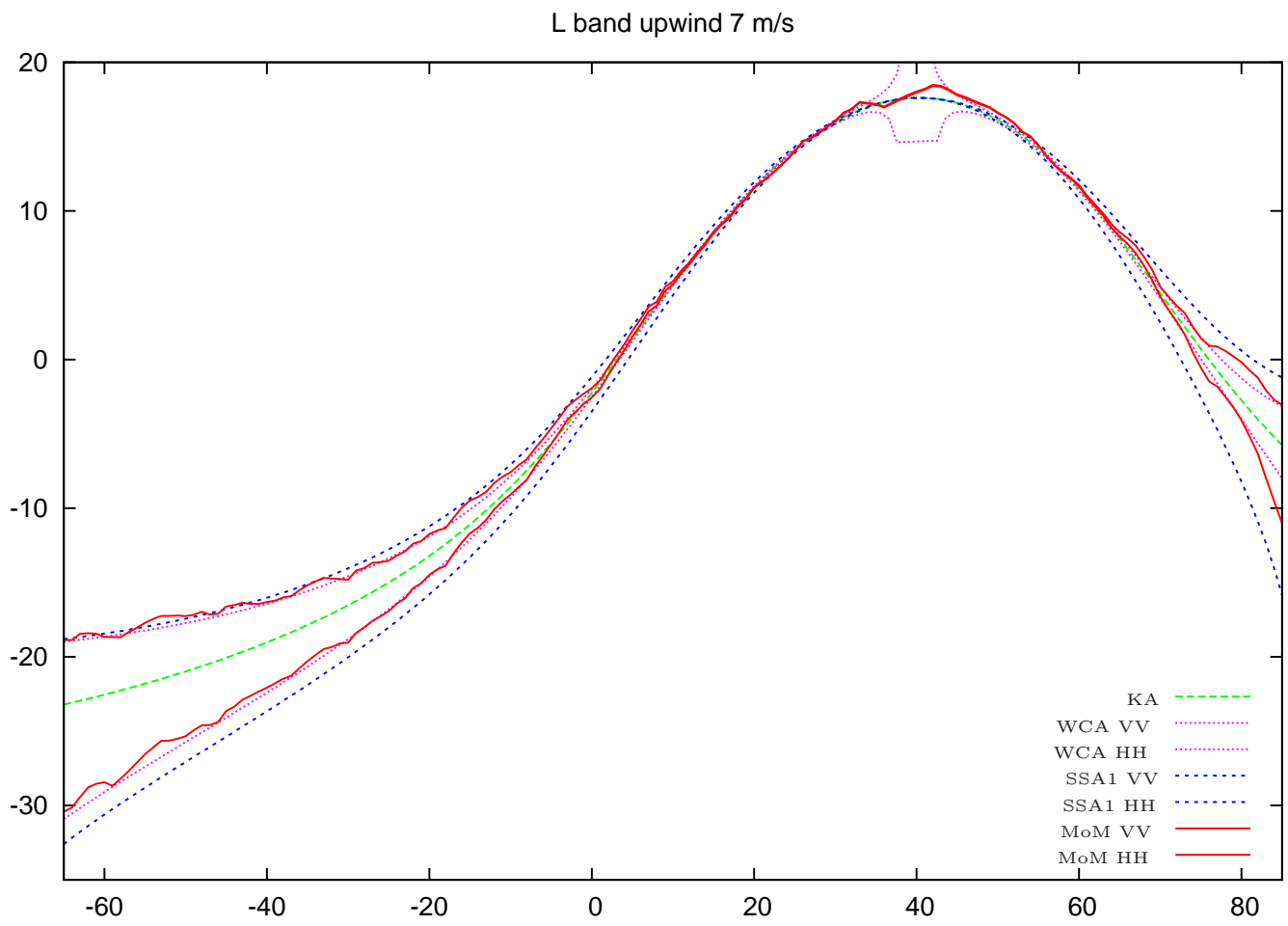

Figure 3: 2D perfectly conducting surfaces, directional Elfouhaily spectrum, $7 \mathrm{~m} / \mathrm{s}$, upwind, L band. Comparison of KA, SSA1 and WCA with the MoM. Bistatic NRCS in the plane of incidence at 40 degrees incidence

\subsection{Comparison with the Two-Scale Model}

In the configurations which are not available for the MoM, it is interesting to compare WCA with the classically employed model in sea surface scattering, namely the Two-Scale Model. We will use the cut-off invariant Two-Scale Model developed recently in [11], which is based on a combination of GO and SSA1 (GO-SSA) and can be symbolically written as:

GO-SSA $=($ Large scales GO $) \times($ Small scales coherent field $)+\mathrm{SSA} 1 *($ facet slopes $)$

Figures 5 and 6 show the monostatic NRCS as a function of the incidence angle at $\mathrm{L}$ and $\mathrm{Ku}$ band by a $7 \mathrm{~m} / \mathrm{s}$ wind speed. WCA and GO-SSA turn out to be extremely close in both polarization over the whole diagram, save for large angles (beyond 60 degrees) in $\mathrm{HH}$ polarization. This fact has been verified for other winds and bands which are not reproduced here. Hence, it appears that the simple combination of KA and Bragg NRCS which is involved in the simplified WCA formulation (4.32) is capable to reproduce a Two-Scale Model, at least numerically. We do not have a complete formal argument to 


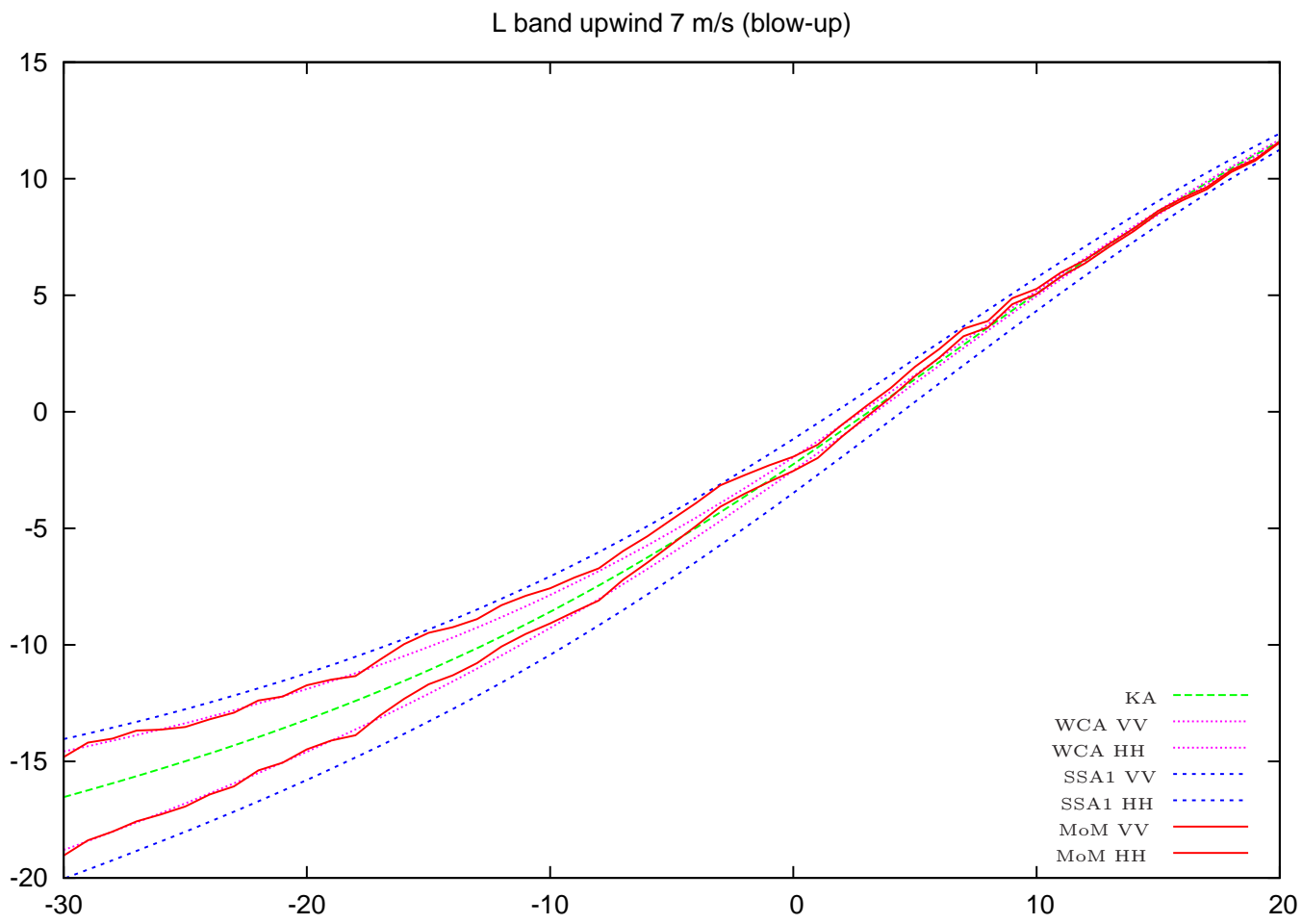

Figure 4: Blow-up of figure 3 at moderate angles

explain the agreement between WCA and GOSSA. An analytical identification is improbable since no tilting effect is introduced in WCA. However, an implicit separation of scales is operated in the first term of (4.32) through the difference of the Kirchhoff integral and the Bragg spectrum. The occurrence of large scales (with respect to the incident wavelength) separates more and more the Kirchhoff integral from its small-scale limiting form. This large scale correction modulates the additional SPM term. Since this correction is polarization independent, it has a relatively higher impact on the HH Bragg component, which is smaller in magnitude than its VV counterpart. This is clearly visible on the plots, where the HH NRCS is enhanced by one or two $\mathrm{dB}$ with respect to SSA1 (which would be close to SPM at large incidences). These numerical results suggest that the "tilting effect" in the GOSSA Two-Scale Model (SSA1* facet slopes instead of SSA1) is far less important than the "separation of scale" effect (large scale GO). The small divergence observed at large angles for the HH NRCS of GOSSA and WCA might be due precisely to this missing tilting effect. 


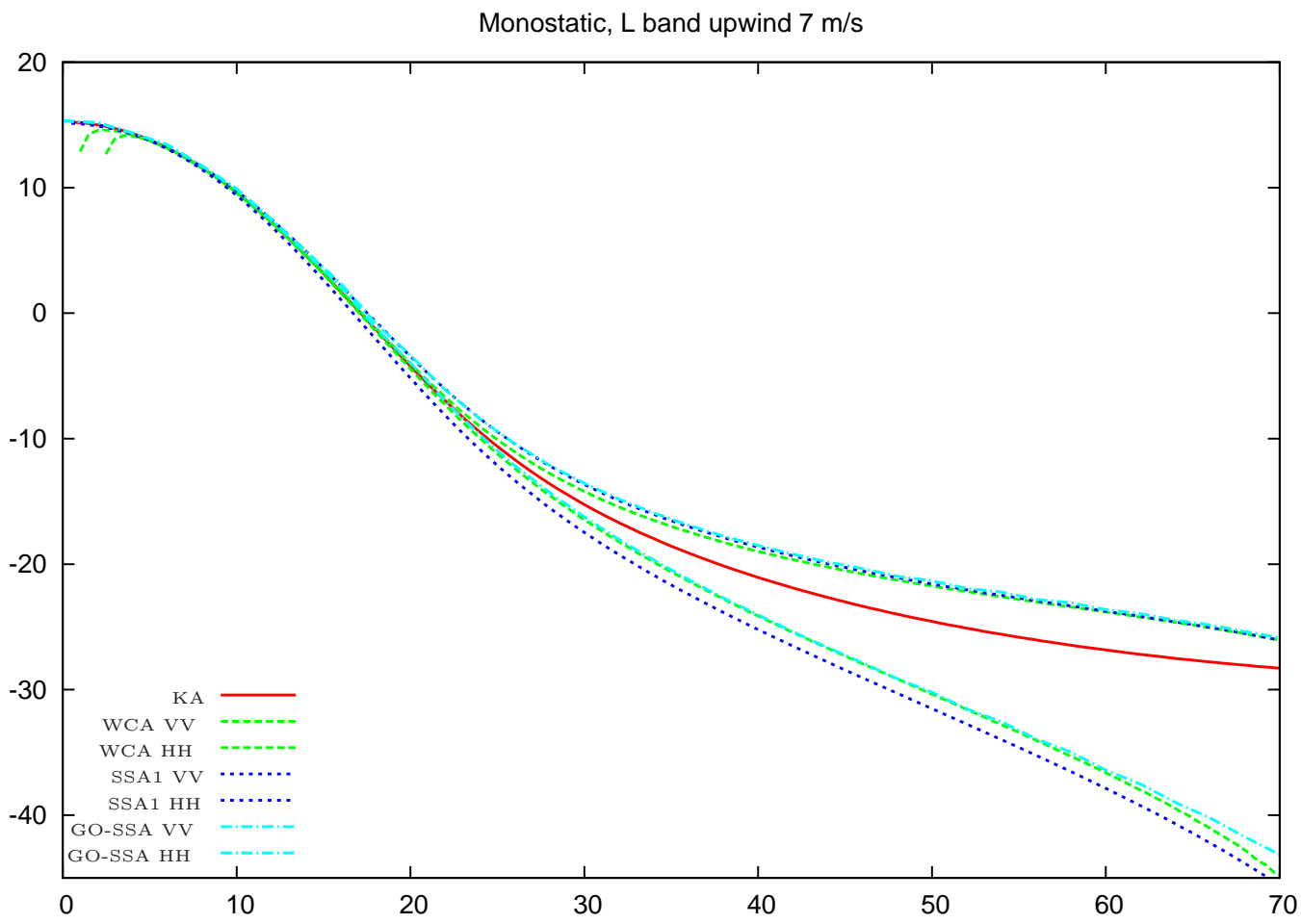

Figure 5: 2D sea surfaces, directional Elfouhaily spectrum, $7 \mathrm{~m} / \mathrm{s}$, upwind, L band, $\varepsilon_{r}=65+i 34$. Comparison of KA, SSA1 and WCA with the GO-SSA. Monostatic NRCS

\section{Conclusion and perspectives}

In spite of many years of research on approximate scattering models, it seems that the historical methods, KA and SPM, remain the unavoidable building blocks of unified models. This is particularly stringent in the case of the sea surface, where the Kirchhoff filtering formula turns any curvature correction into a mere Bragg contribution away from the specular region, resulting in what we have termed the universal WCA. This result has a strong potential in inversion of sea spectra from remote sensing data. In the micro-wave band where the sea conductivity is high, the Kirchhoff dyad is quasi-insensitive to polarization. It follows from the main formula (4.32) that the surface spectrum at the Bragg frequency can be obtained through a simple difference of the co-polarized NRCS:

$$
4 \pi \Gamma\left(\boldsymbol{Q}_{H}\right)=\frac{\sigma_{V V}-\sigma_{H H}}{\left|\mathbb{B}_{V V}\right|^{2}-\left|\mathbb{B}_{H H}\right|^{2}},
$$

a result which is usually expected to hold in the low-frequency SPM regime only. This formula was first proposed on heuristic grounds almost fifteen years 


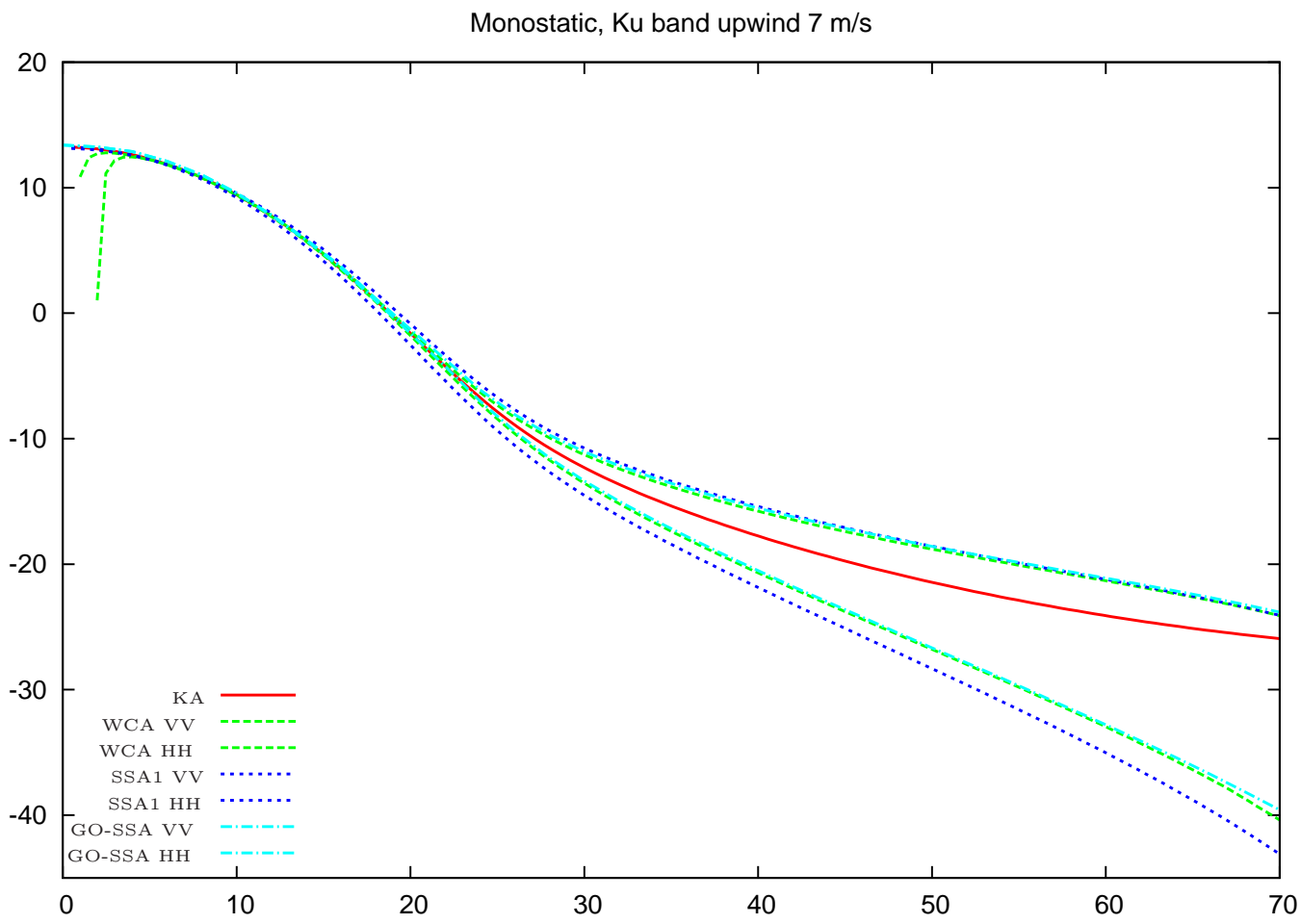

Figure 6: 2D sea surfaces, directional Elfouhaily spectrum, $7 \mathrm{~m} / \mathrm{s}$, upwind, $\mathrm{Ku}$ band, $\varepsilon_{r}=38+i 40$. Comparison of KA, SSA1 and WCA with GO-SSA. Monostatic NRCS

ago by one of the authors [16]. A systematic validation of various sea spectrum models could thus be undertaken on the basis of space remote sensing data.

\section{Appendix}

\subsection{Statistical formulas}

Let $\eta$ be a stationary Gaussian process with correlation function $\rho$ and define $E=\mathrm{e}^{-\mathrm{i} \mathrm{Q}_{z} \eta}$ and $E_{0}=\mathrm{e}^{-\mathrm{i} \mathrm{Q}_{z} \eta_{0}}$. Then

$$
F=\left\langle E E_{0}^{*}\right\rangle=\mathrm{e}^{-Q_{z}\left(\rho_{0}-\rho\right)}
$$

Now let $\mathbb{G}(\boldsymbol{\nabla})[\eta]$ be an operator involving only second-order derivatives of $\eta$ such as $\Delta \eta, \nabla \nabla, e t c \ldots$. Then we have:

$$
\left\langle\mathbb{G}(\boldsymbol{\nabla})[\eta] E E_{0}^{*}\right\rangle=-\mathrm{iQ}_{\mathrm{z}}\left\{(\mathbb{G}(\boldsymbol{\nabla})[\rho])_{0}-\mathbb{G}(\boldsymbol{\nabla})[\rho]\right\} \mathrm{F}
$$


and

$$
\left\langle\mathbb{G}(\boldsymbol{\nabla})[\eta] E\left(\mathbb{G}^{*}(\boldsymbol{\nabla})[\eta]\right)_{0} E_{0}^{*}\right\rangle=\left\{\mathbb{G}^{2}(\boldsymbol{\nabla})[\rho]-Q_{z}^{2}\left|(\mathbb{G}(\boldsymbol{\nabla})[\rho])_{0}-\mathbb{G}(\boldsymbol{\nabla})[\rho]\right|^{2}\right\} F
$$

Proof. Standard manipulations on Gaussian processes.

\subsection{The "Kirchhoff filtering formula" and its consequences}

In the case of sea spectra, one has the following approximation for a Laplacian under a Kirchhoff integral away from the specular region:

$$
\frac{1}{(2 \pi)^{2}} \int \boldsymbol{d} \boldsymbol{r} \mathrm{e}^{-\mathrm{i} \boldsymbol{Q}_{\boldsymbol{H}} \cdot \boldsymbol{r}} \mathrm{e}^{-Q_{z}^{2}\left(\rho_{0}-\rho\right)} \Delta \rho \simeq-Q_{H}^{2} \Gamma\left(\boldsymbol{Q}_{H}\right),
$$

as soon as $Q_{H}>>\sqrt{m s s} Q_{z}$, where $m s s$ is the mean square slope of the surface. More generally, one has

$$
\frac{1}{(2 \pi)^{2}} \int \boldsymbol{d} \boldsymbol{r} \mathrm{e}^{-\mathrm{i} \boldsymbol{Q}_{\boldsymbol{H}} \cdot \boldsymbol{r}} \mathrm{e}^{-Q_{z}^{2}\left(\rho_{0}-\rho\right)} \mathbb{G}(\boldsymbol{\nabla})[\rho]=-\mathbb{G}\left(\boldsymbol{Q}_{H}\right) \Gamma\left(\boldsymbol{Q}_{H}\right)
$$

for any nonlinear kernels with polynomial dependence on slopes. This shows that the curvature contributes to the Kirchhoff integral essentially through its filtered version around the Bragg frequency. By "sea spectrum" we mean any spectrum $(\Gamma)$ which is approximately power-law decreasing beyond some peak wavenumber, together with the omnidirectional slope spectrum $\left(k^{2} \Gamma\right)$.

Proof. Define $F$ as in (8.46), so that its Fourier Transform writes

$$
\widehat{F}\left(\boldsymbol{Q}_{H}\right)=\frac{1}{(2 \pi)^{2}} \int \boldsymbol{d} \boldsymbol{r} \mathrm{e}^{-\mathrm{i} \boldsymbol{Q}_{\boldsymbol{H}} \cdot \boldsymbol{r}} \mathrm{e}^{-Q_{z}^{2}\left(\rho_{0}-\rho\right)}
$$

Then by the convolution theorem

$$
\frac{1}{(2 \pi)^{2}} \int \boldsymbol{d} \boldsymbol{r} \Delta \rho \mathrm{e}^{-\mathrm{i} \boldsymbol{Q}_{\boldsymbol{H}} \cdot \boldsymbol{r}} F(\boldsymbol{r})=-\left(\widehat{F} *\left(k^{2} \Gamma\right)\right)\left(\boldsymbol{Q}_{H}\right),
$$

where the asterisk stands for the convolution product. To estimate this integral, we use a two-scale argument and decompose the spectrum into small and large scales, $\Gamma=\Gamma_{L}+\Gamma_{s}$. We call the $\rho_{L}$ and $\rho_{s}$, respectively, the corresponding correlation functions. For sea spectra, a separation frequency around Bragg wavenumber ensures that $Q_{z}^{2} \rho_{s 0}<<1$, so we may treat the small scales perturbatively in the function $F$ :

$$
\begin{aligned}
\widehat{F}(\boldsymbol{k}) & \simeq \frac{1}{(2 \pi)^{2}} \int \boldsymbol{d} \boldsymbol{r} \mathrm{e}^{-\mathrm{i} \boldsymbol{k} \cdot \boldsymbol{r}} \mathrm{e}^{-Q_{z}^{2}\left(\rho_{L 0}-\rho_{L}\right)}+Q_{z}^{2} \frac{1}{(2 \pi)^{2}} \int \boldsymbol{d} \boldsymbol{r} \rho_{s} \mathrm{e}^{-\mathrm{i} \boldsymbol{k} \cdot \boldsymbol{r}} \mathrm{e}^{-Q_{z}^{2}\left(\rho_{s 0}-\rho_{s}\right)} \\
& =\widehat{F}_{L}(\boldsymbol{k})+Q_{z}^{2}\left(\Gamma_{s} * \widehat{F}_{L}\right)(\boldsymbol{k})
\end{aligned}
$$


where $\widehat{F}_{L}$ is the Fourier integral (8.46) associated to the large scale process $\rho_{L}$. Injecting this last approximation in the convolution integral (8.52) we obtain:

$$
\left(\widehat{F} *\left(k^{2} \Gamma\right)\right)\left(\boldsymbol{Q}_{H}\right) \simeq\left(\widehat{F}_{L} *\left(k^{2} \Gamma\right)\right)\left(\boldsymbol{Q}_{H}\right)+Q_{z}^{2}\left(\Gamma_{s} * \widehat{F}_{L}\right) *\left(k^{2} \Gamma\right)\left(\boldsymbol{Q}_{H}\right)
$$

In the first term, the function $\widehat{F}_{L}$ is the scattering diagram of a large scale process and is thus amenable to a GO approximation, namely a centered Gaussian distibution of variance $m s s_{L} Q_{z}^{2}$, where $m s s_{L}$ is the large scale mean square slope. Hence the first convolution integral involves the slope spectrum $k^{2} \Gamma(\boldsymbol{k})$ only in a small domain around Bragg wavenumber, say $\left\|\boldsymbol{k}-\boldsymbol{Q}_{H}\right\| \leq 2 \sqrt{m s s_{L}} Q_{z}$. Since we have assumed $\sqrt{m s s} Q_{z}<<Q_{H}$, this implies that the ratio $k / Q_{H}$ remains close to unity in the efficient integration domain. Since the function $k^{2} \Gamma$ has essentially a power-law behaviour above the peak number, this means that one can neglect its deviation from its value at the Bragg frequency in the last integral. Hence

$$
\left(\widehat{F}_{L} *\left(k^{2} \Gamma\right)\right)\left(\boldsymbol{Q}_{H}\right) \simeq Q_{H}^{2} \Gamma\left(\boldsymbol{Q}_{H}\right) \int \boldsymbol{d} \boldsymbol{k} \widehat{F}_{L}(\boldsymbol{k})=Q_{H}^{2} \Gamma\left(\boldsymbol{Q}_{H}\right),
$$

where we have used $F_{L}(0)=1$. It remains to show that the remaining term in (8.54) is negligible with respect to the former. To this aim, we use the same GO argument to approximate $\Gamma_{s} * \widehat{F}_{L} \simeq \Gamma_{s}$ and resort again to a two-scale decomposition:

$$
Q_{z}^{2}\left(\Gamma_{s} *\left(k^{2} \Gamma\right)\right)=Q_{z}^{2}\left(\Gamma_{s} *\left(k^{2} \Gamma_{s}\right)\right)+Q_{z}^{2}\left(\Gamma_{s} *\left(k^{2} \Gamma_{L}\right)\right)
$$

Applying Hölder inequality to the respective convolution integrals, we have the crude estimation:

$$
\left|Q_{z}^{2}\left(\Gamma_{s} *\left(k^{2} \Gamma\right)\right)\right| \leq Q_{z}^{2} \rho_{s 0} \sup _{\boldsymbol{k}}\left(k^{2} \Gamma_{s}(\boldsymbol{k})\right)+Q_{z}^{2} m s s_{L} \sup _{\boldsymbol{k}}\left(\Gamma_{s}(\boldsymbol{k})\right)
$$

For sea spectra the functions $k^{2} \Gamma_{s}$ and $\Gamma_{s}$ are decreasing above the separation frequency, so that their maximum is of the order of their value at the Bragg frequency. This makes every term in (8.57) of the order of $Q_{z}^{2} \rho_{s 0} Q_{H}^{2} \Gamma_{s}\left(\boldsymbol{Q}_{H}\right)$ or $Q_{z}^{2} m s s_{L} \Gamma_{s}\left(\boldsymbol{Q}_{H}\right)$, that is in any case negligible with respect to $Q_{H}^{2} \Gamma\left(\boldsymbol{Q}_{H}\right)$. Altogether this proves (8.49). $\square$ The same argument applies to (8.50).

Even though we have used a two-scale argument, which implicitely assumes that the surface has both large- and small-scale components, note that the proof remains valid if the large scale are removed, in which case the filter $\widehat{F}_{L}$ is a perfect Dirac function, or if the small-scale are removed, in which case the demonstration is trivial. Note also that the off-specular region of validity is in fact controlled by the large scale mss rather than the total mss. The latter is, however, a more universal parameter. We also see that the proof not only applies to sea spectra, but in fact to every kind of spectrum which is approximatively power-law beyond the Bragg frequency.

The approximation (8.49) has been tested for an isotropic Elfouhaily spectrum at increasing wind speed $(5,10$ and $15 \mathrm{~m} / \mathrm{s})$ in C-band $(5 \mathrm{GHz})$. Figure 
7 shows the relative error (in percent) as a function of the monostatic angle. The error is larger than $20 \%$ around nadir, which has no consequence since the KA term in WCA dominates in this region (see the discussion below). For larger angles, the quality of the approximation increases rapidly, with an error less than $5 \%$ above 45 degrees. We have checked that this accuracy is quite insensitive to the incident frequency, and very similar results are obtained in $\mathrm{L}$ or X band.

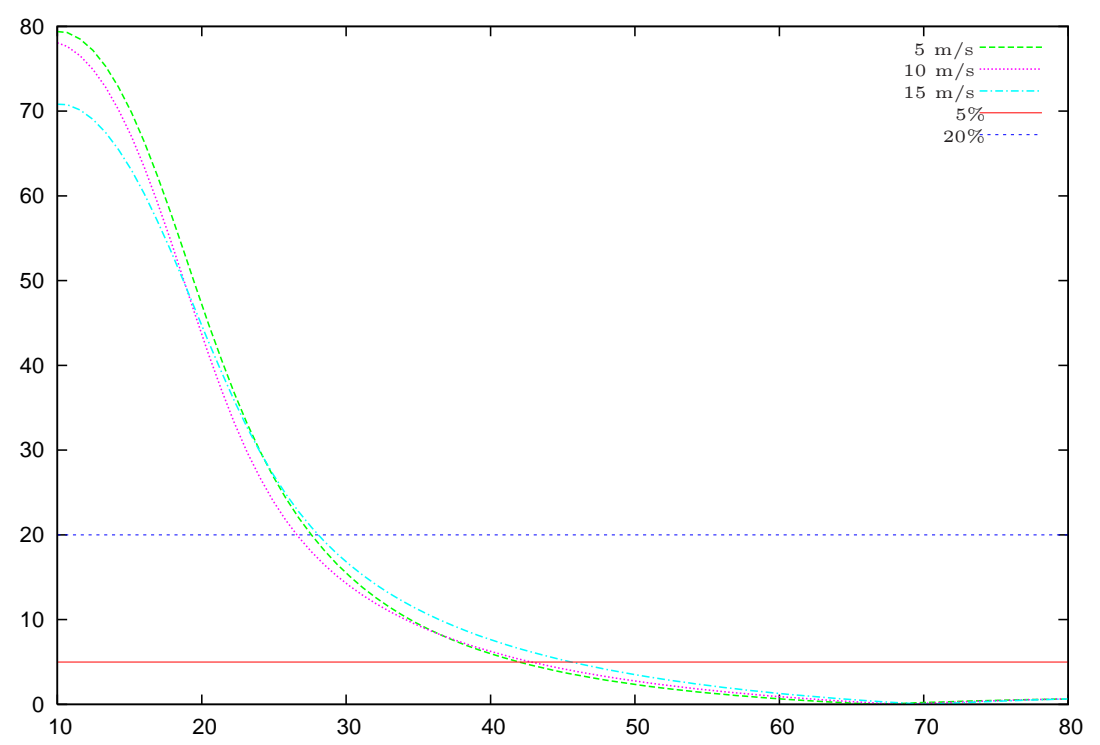

Figure 7: Relative error (in percent) in the Kirchhoff filtering formula for an omnidirectional Elfouhaily spectrum at different wind speeds

Figure 8 displays a comparison of the "true" NRCS (4.26-4.27) for the Laplacian kernel (2.15) and its universal approximation (4.32) on a typical test case, namely the C-Band ( $5 \mathrm{GHz}, \varepsilon_{r}=65+34 \mathrm{i}$ ) backscattering NRCS for an omnidirectional Elfouhaily spectrum ([17]) at $10 \mathrm{~m} / \mathrm{s}$ wind speed. The two co-polarized components are plotted on the same graph, with the vertical (resp. horizontal) polarization in the upper (resp. lower) set of curves. The (unpolarized) KA NRCS is given for reference. The numerical approximation (4.32) is excellent beyond 5 degrees off the nadir direction (the Kirchhoff integral with Laplacian is numerically difficult to evaluate around nadir, as witnessed by the small divergence on the graph in this region). Even through the accuracy of the Kirchhoff filtering formula (8.49) is poor at small angles (below 30 degrees), the overall accuracy of the universal WCA is very good because the KA term is dominant in the near specular region. A small difference is, however, observed at large $\mathrm{HH}$ angles. We put this on the account of limited accuracy in the computation 
of the Kirchhoff integral with Laplacian kernel.

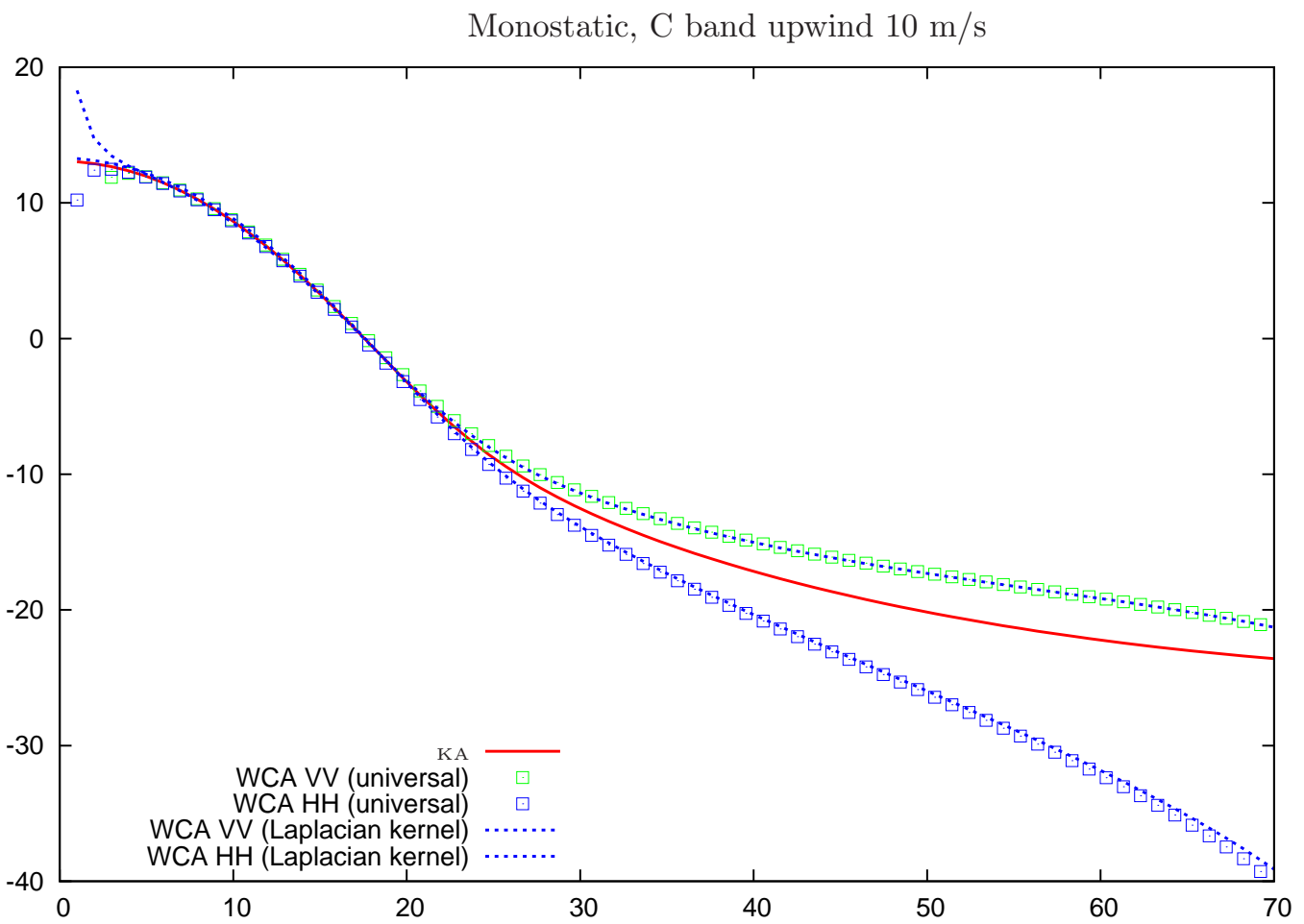

Figure 8: Validity of the Kirchhoff filtering formula for the WCA NRCS

Acknowledgements: This work was supported by the ANR project no. ANR-09-BLAN-0232-01 SIMODE.

\section{References}

[1] T.M. Elfouhaily and C.A. Guérin. A critical survey of approximate scattering wave theories from random rough surfaces. Waves in Random and Complex Media, 14(4):1-40, 2004.

[2] R. Dashen and D. Wurmser. A new theory for scattering from a surface. J. Math. Phys., 32(4):971-985, 1991.

[3] R. Dashen and D. Wurmser. Approximate representations of the scattering amplitude. J. Math. Phys., 32(4):986-996, 1991.

[4] R. Dashen and D. Wurmser. Applications of the new scattering formalism: the dirichlet boundary condition. J. Math. Phys., 32(4):997-1003, 1991. 
[5] AA Mouche, B. Chapron, and N. Reul. A simplified asymptotic theory for ocean surface electromagnetic wave scattering. Waves in Random and Complex Media, 17(3):321-341, 2007.

[6] AA Mouche, B. Chapron, N. Reul, D. Hauser, and Y. Quilfen. Importance of the sea surface curvature to interpret the normalized radar cross section (DOI 10.1029/2006JC004010). J. Geophys. Res., 112(C10):10002, 2007.

[7] AA Mouche, B. Chapron, N. Reul, and F. Collard. Predicted Doppler shifts induced by ocean surface wave displacements using asymptotic electromagnetic wave scattering theories. Waves in Random and Complex Media, 18(1):185-196, 2008.

[8] T. Elfouhaily, S. Guignard, R. Awadallah, and D.R. Thompson. Local and non-local curvature approximation: a new asymptotic theory for wave scattering. Waves in Random and Complex Media, 13(4):321-337, 2003.

[9] C.A. Guérin, G. Soriano, and T. Elfouhaily. Weighted curvature approximation: numerical tests for 2D dielectric surfaces. Waves in Random and Complex Media, 14(3):349-363, 2004.

[10] C. Bourlier, N. Dechamps, and G. Berginc. Comparison of asymptotic backscattering models (SSA, WCA, and LCA) from one-dimensional Gaussian ocean-like surfaces. IEEE Transactions on Antennas and Propagation, 53(5):1640-1652, 2005.

[11] G. Soriano and C.A. Guérin. A cutoff invariant two-scale model in electromagnetic scattering from sea surfaces. IEEE Geoscience and Remote Sensing Letters, 5(2):199-203, 2008.

[12] A. G. Voronovich. Small-slope approximation for electromagnetic wave scattering at a rough interface of two dielectric half-spaces. Waves in Random Media, 4:337-367, 1994.

[13] A. G. Voronovich and V. U. Zavorotny. Theoretical model for scattering of radar signals in $\mathrm{Ku}$ - and $\mathrm{C}$-bands from a rough sea surface with breaking waves. Waves in Random Media, 11:247-269, 2001.

[14] G. Soriano and M. Saillard. Progress In Electromagnetic Research X, chapter Modelization of the scattering of electromagnetic waves from the ocean surface, pages 102-128. EMW Publishing 2003, 2003.

[15] M. Saillard and G. Soriano. Fast numerical solution for scattering from rough surfaces with small slopes. IEEE Trans. Antennas Propag., 52:27992802,2004 .

[16] B. Chapron, V. Kerbaol, and D. Vandemark. A note on the reslationships between sea surface roughness and microwave polarimetric backscatter measurements results. In Proceedings on International Workshop Polrad'96, pages WPP-135. ESA, 1997. 
[17] T. Elfouhaily, B. Chapron, K. Katsaros, and D. Vandemark. A unified directional spectrum for long and short wind-driven waves. J. Geophys. Res., 102(C7):15,781-15,796, July 1997. 